\title{
Occurrence of Chlamydia trachomatis and Chlamydia pneumoniae in paediatric respiratory infections
}

\author{
W.C. Webley*, Y. Tilahun*, K. Lay*, K. Patel*, E.S. Stuart*, \\ C. Andrzejewski ${ }^{\#}$ and P.S. Salva
}

ABSTRACT: An emerging body of evidence suggests that half of asthma in both children and adults is associated with chronic lung infection. The aim of the present study was to determine the frequency of viable Chlamydia pneumoniae $(C p)$ and $C$. trachomatis $(C t)$ in the respiratory tracts of paediatric patients with chronic respiratory diseases.

Bronchoalveolar lavage fluid (BALF) samples obtained from 182 children undergoing bronchoscopy for clinical reasons were assayed using PCR analysis, in vitro tissue culture and immunofluorescence staining for the presence of $\mathrm{Cp}$ and $\mathrm{Ct}$.

Chlamydia-specific DNA was detected by PCR in 124 (68\%) out of 182 patients; 79 were positive for $\mathrm{Cp}, 77$ positive for $\mathrm{Ct}$ and 32 for both organisms; 75 patients had cultivable Chlamydia. Ct DNA prevalence decreased, whereas $C p$ positivity generally increased with age. A total of 59 out of 128 asthma patients and 16 out of 54 nonasthmatics were Chlamydia culture positive. When the patients were divided into inflammatory versus noninflammatory airway disease, there were 69 (46\%) out of 150 and six (18\%) out of 32 BALF samples with cultivable Chlamydia, respectively.

Viable Chlamydia pneumoniae and Chlamydia trachomatis occur frequently in children with chronic respiratory diseases and may be more prevalent in asthma patients. To the current authors' knowledge, this is the first report of viable Chlamydia trachomatis in the lungs of children.

KEYWORDS: Asthma, Chlamydia, chronic, lung disease

$\mathbf{R}$ espiratory diseases represent a major cause of disability and mortality among all age groups and races worldwide. They are also a leading cause of hospitalisation and morbidity in both adults and children, especially in developing countries [1-3]. Chronic respiratory diseases, which include asthma and chronic obstructive pulmonary disease (COPD), are just as prevalent as acute forms of these disorders [2]. In addition to known environmental and genetic contributors, there is growing evidence that pathogenic micro-organisms may play a role in diseases such as asthma [4-6]. It is often difficult, however, to identify the aetiological agent(s) of these respiratory infections, owing to a lack of standard diagnostic tools and the need for invasive procedures, such as lung aspirations or pulmonary biopsies, in order to confirm diagnosis. The worldwide increase in the incidence of asthma and the impact of the disease on public health, however, has led to renewed interest and investigations into its aetiopathogenesis.
Chlamydia trachomatis $(C t)$ and C. pneumoniae $(C p)$ are two of the most common members of the Chlamydiaceae family that infect humans. $C p$ is thought to be responsible for $10-15 \%$ of community-acquired pneumonia and 5\% of pharyngitis and sinusitis cases [7, 8]. Approximately $50 \%$ of healthy young adults and $75 \%$ of elderly persons have serological evidence of previous $C p$ infection [9]. There is increasing evidence that $C p$ may play a role in paediatric asthma onset as well as in possible exacerbations of asthmatic symptoms [10, 11]. Conversely, $\mathrm{C} t$ has been recognised as a pathogen in nongonococcal urethritis, salpingitis, endocervicitis, pelvic inflammatory disease, inclusion conjunctivitis of neonates, follicular conjunctivitis of adults, infantile pneumonia and associated diseases [12, 13]. Vertical transmission of infection from mother to the infant may result in the development of conjunctivitis and pneumonia $[14,15]$. In the past, multiple investigations of paediatric pneumonia have emphasised the

\section{AFFILIATIONS}

*Dept of Microbiology, University of Massachusetts, Amherst,

\#Depts of Pathology, and "Paediatric Pulmonology, Baystate Medical Center, Springfield, MA USA.

\section{CORRESPONDENCE}

W.C. Webley

University of Massachusetts

Dept of Microbiology

Morrill Science Center IVN Rm 203 Amherst

MA 01003

USA

Fax: 14135451578

E-mail: wilmore@

microbio.umass.edu

Received:

February 082008

Accepted after revision:

October 012008

STATEMENT OF INTEREST

None declared.

European Respiratory Journal Print ISSN 0903-1936

Online ISSN 1399-3003 
importance of infections with $C t$ in infants between 2 weeks and 4 months of age [16-18]. While stringent prenatal screening in the USA has greatly reduced the number of cases of neonatal conjunctivitis, there have been reports that ocular prophylaxis can fail to prevent neonatal chlamydial conjunctivitis and does not prevent colonisation or infection at other sites, such as the lungs [3, 19]. Approximately $5-22 \%$ of pregnant women are thought to have $C t$ infection of the cervix, and $30-50 \%$ of neonates born to infected mothers show culture evidence of infection. Of infected neonates, $15-25 \%$ present with clinical conjunctivitis and nasopharyngitis that in some cases develops into neonatal pneumonitis [9]. Published reports have documented that many infants infected with $C t$ at birth remain infected for months or years in the absence of specific antimicrobial therapy [20]. Indeed, some reports have suggested that wheezing may be another clinical expression of $C t$ infection and that this organism should be routinely assayed for in children who wheeze but have no demonstrable allergy and do not respond to the usual anti-asthmatic medications [21]. The current authors recently confirmed the presence of Chlamydia in bronchoalveolar lavage fluid (BALF) samples from paediatric patients with various chronic respiratory diseases using PCR and tissue culture techniques [22]. In the work presented herein, using established species specific PCR and culture techniques, these investigations were extended to an examination of the prevalence of both $C p$ and $C t$ in BALF samples collected from paediatric patients. It was shown that both infectious $C p$ and $C t$ are frequently present in lung washings from children with chronic respiratory diseases, and that both organisms may contribute to Chlamydia-mediated pneumonitis.

\section{MATERIALS AND METHODS Specimens}

A prospective, consecutive, noninterventional cohort analysis of patients with various pulmonary disorders undergoing elective diagnostic bronchoscopy with BALF sample collection was conducted in a group of 184 patients from a communitybased/academic hospital setting. Of these specimens, 70 were previously studied and reported on in a prior communication [22]. Two patients aged $>20$ yrs were excluded from the study because of the inclusion criteria of age $\leqslant 20 \mathrm{yrs}$ for this paediatric cohort based on American Academy of Paediatrics accepted criteria [23]. Patients were recommended for bronchoscopy because they all met the criteria of having severe, persistent airway disease that was nonresponsive to therapy. Residual BALF samples obtained from study participants were de-identified in a manner compliant with the Health Insurance Portability and Accountability Act, and given alphanumeric codes prior to laboratory analysis. Approval for the study was obtained from the Institutional Review Board at Baystate Medical Center (Springfield, MA, USA). Written informed consent was obtained from the guardian of each patient prior to inclusion in the study and patients were not contacted during the course of the study, nor were they made aware of the results of the investigation. BALF was collected as previously reported $[22,24]$.

\section{PCR analysis}

Genomic DNA was isolated from BALF samples and PCR performed as previously described [22]. Initially, isolated DNA was amplified using a $16 \mathrm{~S}$ signature sequence to detect all strains of Chlamydiales, as previously reported [22, 25]. Cp-specific PCR was performed using the previously published primer pair Cpn 201 and 202 to generate a 207-bp product [26], while Ct-specific PCR was performed using the P1 and omp2 primer set that amplified a 1,100-bp segment of the omp1 gene [27]. The PCR products were separated by electrophoresis on a $2 \%$ agarose gel and visualised by staining with ethidium bromide. Photographs were taken with the Syngene GeneFlash gel documentation system (Syngene USA, Frederick, MD, USA).

\section{Culture}

Cells from BALF samples were pelleted, rinsed with sterile phosphate buffered saline and lysed with sterile glass beads in sucrose phosphate glutamate buffer. Cultures were performed as previously described [22] and cells were stained with a 1:100 dilution of a rabbit anti-Chlamydia antibody (BIODESIGN International, Saco, ME, USA) and visualised with a 1:1,000 dilution of Alexa-Fluor 488 goat anti-rabbit secondary antibody (Invitrogen, Molecular Probes, Carlsbad, CA, USA). The slides were then examined and photographs taken using a Zeiss LSM 510 Meta Confocal System (Carl Zeiss MicroImaging, Inc., Thornwood, NY, USA).

\section{BALF cell counts}

Upon recovery, BALF cell counts and differentials were performed according to standard techniques in the haematology clinical laboratory at the Baystate Medical Center. Cell enumeration was performed manually using a cell counting chamber (haemocytometer of Improved Neubauer type; Fisher Scientific, Pittsburgh, PA, USA) under phase microscopy with results expressed as number of cells per cubic millimetre. BALF differential counts were performed using Wright stained cytospin preparations of BALF which were then examined under oil immersion microscopy $(50 \times$ or $100 \times$ magnification $)$. Results were expressed on the basis of a 100-cell count survey.

\section{Statistics}

Cross-tabs with the Fisher's exact test and Chi-squared test were used to determine significance. Univariate and bivariate analyses as well as logistic regression were also used to establish interactions between and among variables. For all analyses, tests were two sided and the level of significance was $p \leqslant 0.05$.

\section{RESULTS}

\section{Patient demographics}

The cohort of paediatric respiratory disease patients in the present study consisted of 100 males and 82 females. The average age of this group of patients was 8.7 yrs. Patients were from four different ethnic groups as follows: 121 white patients, 45 Hispanic patients, 15 black patients and one patient of Asian descent (table 1). Asthma diagnosis was made by a combination of family or personal history of atopy, elevated total immunoglobulin (Ig)E levels, positive skin or radioallergosorbent test, reversible flow limitation on spirometry, the presence of increased eosinophil levels, basement membrane thickening on bronchial biopsy, or positive methacholine challenge. All asthma patients met the definition of having severe persistent disease that was uncontrolled (according to Global Initiative for Asthma guidelines [28, 


\begin{tabular}{|c|c|c|c|}
\hline Description & Asthma cohort & Nonasthma subcohort & Total \\
\hline Average age yrs & 8.7 & 12.5 & 8.7 \\
\hline \multicolumn{4}{|l|}{ Age range yrs } \\
\hline $0.0-2.0$ & 11 & 18 & 29 \\
\hline $5.1-10.0$ & 41 & 5 & 46 \\
\hline $10.1-15.0$ & 33 & 13 & 46 \\
\hline $15.1-17.0$ & 16 & 5 & 21 \\
\hline \multicolumn{4}{|l|}{ Sex } \\
\hline Male & 68 & 32 & 100 \\
\hline Female & 60 & 22 & 82 \\
\hline Asian & 0 & 1 & 1 \\
\hline \multicolumn{4}{|l|}{ Medication } \\
\hline Yes & 95 & 31 & 126 \\
\hline No & 33 & 23 & 56 \\
\hline
\end{tabular}

Data are presented as $n$, unless otherwise stated. The cohort consisted of 182 patients (100 males and 82 females). There were significant differences in the number of asthma versus nonasthma subjects in the $0-2.0$ yrs age range (Fisher's exact test, $p=0.001$ ), and the $5.1-10.0$ yrs age range ( $p=0.008)$. There were significantly more patients in the nonasthma cohort in the 0-2.0 yrs age range than in the asthma cohort. Conversely, there were significantly more asthma patients in the 5.1-10.0 yrs age group than in the nonasthma cohort. No other age range, sex, ethnicity or medication use category had significant differences between the asthma and nonasthma cohorts.

29]). A diagnosis of asthma was confirmed in 128 (70\%) out of 182 patients. Black patients were diagnosed with asthma more frequently (13 out of 15) compared with other ethnic groups (Hispanic patients: 30 out of 45; white patients: 85 out of 121). The lone Asian patient was nonasthmatic. Nonasthmatic disorders were gastro-oesophageal reflux disease (GERD), aspiration, bronchitis, bacterial bronchitis, structural anomalies (tracheomalacia, large-airway bronchomalacia and minor anatomic variants: accessory bronchi, tracheal bronchus and pinhole bronchus), chronic cough and vascular compression (innominate artery and pulmonary artery compression of left $\begin{array}{lllllllllllll}1 & 2 & 3 & 4 & 5 & 6 & 7 & 8 & 9 & 10 & \mathrm{~L} & 11 & \mathrm{~N} \\ 1213141516 & \mathrm{Ct} & \mathrm{Cp}\end{array}$ $269 \mathrm{bp}$

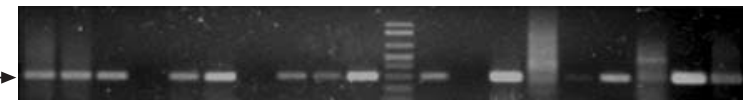

b)
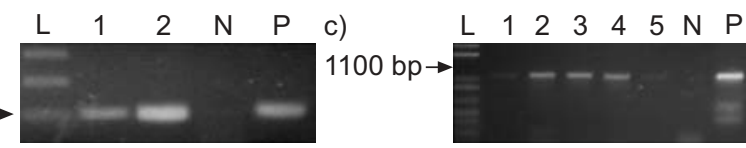

$207 \mathrm{bp}$

FIGURE 1. Representative agarose gels showing Chlamydia PCR products. a) 16S amplified DNA from both Chlamydia-positive (samples 1-3, 5, 6, 8-10, 11-13, 15 and 16) and -negative $(4,7,14)$ bronchoalveolar lavage fluid (BALF) samples compared with the positive controls (for Chlamydia trachomatis $(C t)$ and $\mathrm{C}$. pneumoniae $(C p)$ ) and the negative control $(\mathrm{N})$. b) $\mathrm{Cp}$ primers used to specifically amplify a 207-bp product and c) a Ct-specific primer pair used to amplify a 1,100bp product from the BALF of paediatric patients tested. P: positive control; L: DNA ladder. mainstem artery), cystic fibrosis and recurrent pneumonia of unknown aetiology. Most of the diagnosed asthma patients also displayed GERD and bronchitis. There was no significant relationship between race or sex and BALF culture positivity for the infectious form of Chlamydia. Of the 182 patients, 126 were taking one or more medication(s) at the time of testing, including four patients on antibiotics (amoxicillin, Zithromax, trimethoprim-sulfamethoxazole (TMP-SMX), Cefdinir and Bactrim).

\section{Detection of Chlamydia in BALF}

PCR was performed on the BALF samples using a $16 \mathrm{~S}$ signature ribosomal DNA sequence to determine the prevalence of chlamydial DNA carriage in these samples. PCR amplification of the target sequence resulted in a 298-bp product which was identified by electrophoresis. The data revealed that $124(68 \%)$ patients were positive for the presence of chlamydial DNA (fig. 1a). The current authors have previously reported on the frequency of $C p$ in a similar cohort but did not specifically test all the samples for the presence of $C t$ [22]. This decision was mainly due to the fact that $C t$ is not routinely reported in association with lung infections or pneumonitis; rather, it is mainly observed in sexually transmitted infection cases, as well as conjunctivitis. With strong evidence from the literature that $\mathrm{Ct}$-mediated pneumonitis is possible, especially in neonates [3, 12], all BALF samples that tested positive for chlamydial DNA using the $16 \mathrm{~S}$ primers were re-tested with both $\mathrm{C} p$ - and $\mathrm{Ct}$-specific primers in order to determine the frequency of lung infection with these 
TABLE 2 Frequency of Chlamydia in asthma versus nonasthma patient groups and with corticosteroid administration

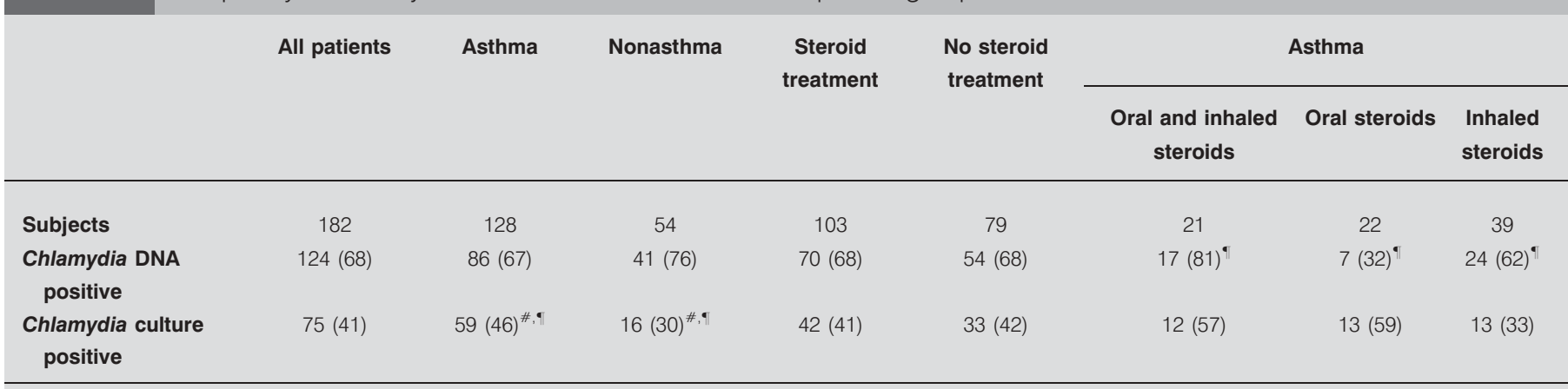

Data are presented as $\mathrm{n}$ or $\mathrm{n}(\%) .{ }^{\#}: \mathrm{p}=0.048$ for asthma versus nonasthma (Fisher's exact test); $"$ : logistic regression confirmed association of asthma and culture positive $($ asthma $(y / n)=$ culture $(y / n)+$ steroids $(y / n)+($ culture $x$ steroids (interaction)); $p=0.033$ for asthma; $p=0.3171$ for steroids; $p=0.3945$ for interaction)

two human pathogens. Of the 182 paediatric patient samples assayed, $79(43.4 \%)$ were positive for the presence of $\mathrm{Cp}$ specific DNA, while $77(42.3 \%)$ were positive for Ct-specific DNA (fig. $1 b$ and c). BALF samples from 32 (17.6\%) paediatric patients contained both $C p$ and $C t$ DNA.

Having recovered both $C t$ and $C p$ DNA at high rates in these BALF samples, it was next attempted to determine the proportion of these organisms that were cultivable at the time of collection. All BALF samples were cultured on human or mouse macrophage cells using previously published protocols [22]. The results revealed that $75(41 \%)$ of the 182 patient samples were positive for Chlamydia when the BALF was cultured (table 2). Since all culture-positive samples were also PCR positive, it is clear that $60 \%$ (75 out of 124) of all PCRpositive samples contained cultivable organisms. There was no significant difference in the finding of infectious Chlamydia between sexes; 33 of the BALF culture-positive samples were from female patients and 42 were from males. However, cultivable chlamydial organisms were found more frequently in the asthmatic population; 59 out of 128 asthmatics versus 16 out of 54 nonasthmatics were culture positive $(p=0.048$, Fisher's exact test; table 2). As an internal control, the patient cohort was divided into inflammatory respiratory disease $(n=150)$ and noninflammatory airway diseases $(n=32)$. The noninflammatory respiratory disease group was categorised as such based on normal bronchial biopsy and normal BALF. Aerobic cultures were also negative for this group, which consisted of structural defects, IgG and IgA deficiencies, aspirations and GERD; the inflammatory group included asthma, bronchitis and pneumonia, and were also categorised based on the bronchial biopsy, BALF and aerobic cultures. When assessed for cultivable Chlamydia, 69 (46\%) of the inflammatory respiratory disease patients harboured cultivable Chlamydia, versus six (18\%) of the noninflammatory disease group (Fisher's exact test (two-sided), $p=0.005$ ). There was no significant association between these groups and the detection of chlamydial DNA (table 3). Evaluation of total serum IgE levels revealed no statistically significant association between patients with elevated serum $\operatorname{IgE}$ and the presence of Chlamydia DNA or cultivable organisms (table 3).

Out of the 182 patients in the paediatric cohort, 103 (56.6\%) were taking some form of steroid therapy at the time of BALF sample collection. In this cohort, $82(64 \%)$ out of the 128 asthmatics were being prescribed corticosteroids; 23 nonasthmatics diagnosed with one or more of the other chronic respiratory diseases listed previously were also being prescribed corticosteroids at the time of sample collection. Of the 82 asthmatic patients taking corticosteroids, 52 (63\%) were positive for the presence of chlamydial DNA and 38 tested positive for cultivable Chlamydia in culture. A total of 18 (78\%) of the 23 nonasthma patients being administered corticosteroids were also positive for chlamydial DNA; five of these were Chlamydia culture positive. Asthma patients being prescribed both oral and inhaled corticosteroids were more likely to

TABLE 3 Prevalence of Chlamydia in inflammatory and noninflammatory airway disease

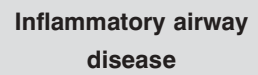

150
$69(46)^{\#}$
$04(69.3)$

Noninflammatory airway disease
Elevated serum IgE Normal serum IgE

32

$6(18)^{\#}$

72
$43(60)$
$26(36)$

$43(60)$

$26(36)$
110

81 (74) $49(45)$

Data are presented as $n$ or $n$ (\%). Inflammatory airway disease included asthma, pneumonia and bacterial bronchitis; noninflammatory diseases included gastrooesophageal reflux disease, aspirations, laryngomalacia, dyspnoea, structural anomalies, chronic cough and vascular compression. * : $\mathrm{p}=0.005$, Fisher's exact test (two sided). There was no significant association with PCR-positive samples $(p=0.531)$. There was no statistically significant association between patients with elevated serum immunoglobulin (Ig)E and the presence of Chlamydia DNA or cultivable organisms. 


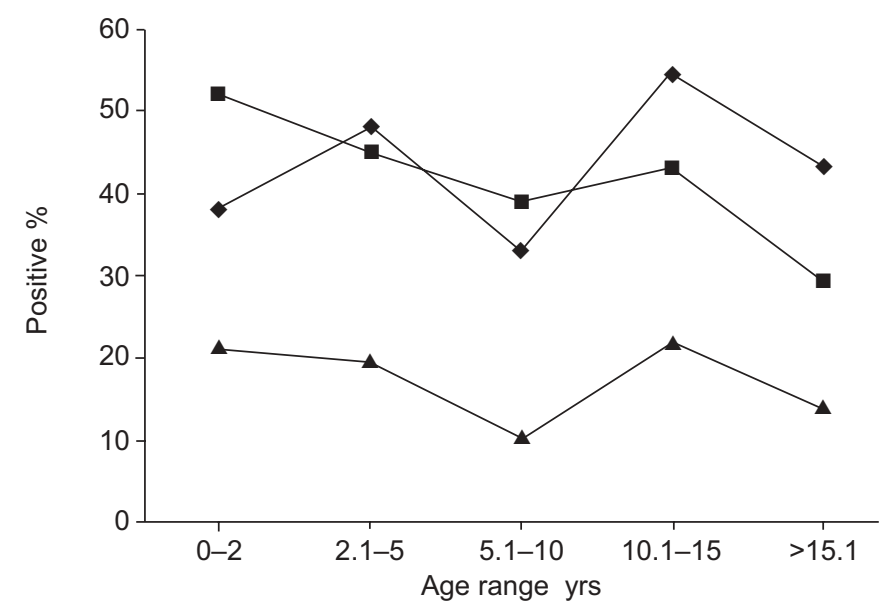

FIGURE 2. Age-based prevalence of chlamydial DNA in bronchoalveolar lavage fluid. The correlation between age and the prevalence of Chlamydia trachomatis $(C t)$ and $C$. pneumoniae $(C p)$ organisms determined by PCR analysis is shown. Percentages were calculated based on the number of patients positive for the particular organism and the total number of patients in that group. Note that patients in the 10.1-15 yrs age range accounted for the highest percentage (35 (76\%) out of 46 ) of Chlamydia DNA-positive patients. The $0-2$ yrs age group harboured the highest percentage of $\mathrm{Ct}$ organisms (15 (51.7\%) out of 29). $\bullet: C p$; 口: $C t ; \mathbf{\Lambda}: C t$ and $C p$. See also table 4.

harbour chlamydial DNA in their BALF (17 out of 21 ) than those taking only oral (seven out of 22; $\mathrm{p}=0.0461$, Fisher's exact test) or inhaled corticosteroids (24 out of 39; $\mathrm{p}=0.133$; table 2 ).

In addition to corticosteroids, four of these patients were being prescribed antibiotics at the time of sample collection. Three of these four patients were diagnosed with asthma (one patient was diagnosed with both asthma and bronchitis) and one patient had bronchitis. The BALF from two of these patients taking Bactrim and TMP-SMX harboured chlamydial DNA and one was culture positive for Chlamydia; patients on amoxicillin and Zithromax were both negative for Chlamydia.

\section{Chlamydia, age and disease associations}

The cohort of 182 respiratory disease patients consisted of 128 (70\%) diagnosed asthmatics. Chlamydial DNA was recovered by PCR amplification from $86(67 \%)$ out of 128 patients diagnosed with asthma (table 2). The remaining 41 patients were Chlamydia negative by PCR. Chlamydial DNA was, however, not exclusively found in the asthma population, with $41(76 \%)$ out of 54 nonasthma patient samples also PCR positive. Of the 32 patients who harboured both $C t$ and $C p$ DNA in their lungs, 20 out of 128 were asthmatic and 12 out of 54 were nonasthmatic. Patients in the $10.1-15$ yrs age range accounted for the highest prevalence (35 (76\%) out of 46 ) of Chlamydia infection (fig. 2 and table 4). The $0-2$ yrs age group harboured the highest percentage of Ct DNA samples (15 $(51.7 \%)$ out of 29$)$. The percentage of patient samples testing positive for C $p$ DNA increased from 11 (38\%) out of 29 in the 02 yrs age group to $19(47.5 \%)$ out of 40 in the $2.1-5$ yrs age range. The number of patients testing positive for the presence of $C t$ DNA decreased over time with increasing age, while the inverse was generally true for $C p$. The prevalence of both $C p$ and $C t$ decreased after age 15 yrs (fig. 2 and table 4).

\begin{tabular}{|c|c|c|c|c|c|c|}
\hline \multirow[t]{3}{*}{ TABLE 4} & \multicolumn{6}{|c|}{$\begin{array}{l}\text { Age-based prevalence of chlamydial DNA in } \\
\text { bronchoalveolar lavage fluid: correlation } \\
\text { between age and the prevalence of Chlamydia } \\
\text { trachomatis }(C t) \text { and C. pneumoniae }(C p) \\
\text { organisms determined by PCR analysis }\end{array}$} \\
\hline & & \multicolumn{5}{|c|}{ Age range yrs } \\
\hline & & $0-2$ & $2.1-5$ & $5.1-10$ & $10.1-15$ & $>15.1$ \\
\hline \multicolumn{2}{|c|}{ Total patients } & 29 & 40 & 46 & 46 & 21 \\
\hline \multicolumn{2}{|c|}{ Total PCR positive } & $20(69)$ & $29(73)$ & $28(61)$ & $35(76)$ & $12(57)$ \\
\hline \multicolumn{2}{|c|}{ Ct PCR positive } & $15(52)$ & $18(45)$ & $18(39)$ & $20(43)$ & $6(29)$ \\
\hline \multicolumn{2}{|c|}{ Cp PCR positive } & $11(38)$ & $19(48)$ & $15(33)$ & $25(54)$ & $9(43)$ \\
\hline \multicolumn{2}{|c|}{$C t$ and $C p$ positive } & $6(21)$ & $8(20)$ & $5(11)$ & $10(22)$ & $3(14)$ \\
\hline \multicolumn{2}{|c|}{ Culture positive } & $14(48)$ & $14(35)$ & $18(39)$ & $20(43)$ & $9(43)$ \\
\hline
\end{tabular}

Data are present as $n$ or $n(\%)$. Percentages are calculated based on the number of patients positive for the particular organism and the total number of patients in that group. Note that patients in the 10.1-15 yr age range accounted for the highest percentage (35 (76\%) out of 46$)$ of Chlamydia DNA-positive patients. The 0-2 yr age group harboured the highest percentage of $\mathrm{Ct}$ organisms (15 (51.7\%) out of 29). See also figure 2.

\section{BALF cellularity and Chlamydia}

BALF collected from each patient was analysed in a clinical setting for the presence of various cell types. Specifically, cell counts were performed in order to determine the number of lipid-laden macrophages (LLMs)/monocytes, eosinophils, lymphocytes and neutrophils. A total of 45 patient samples showed elevated levels of eosinophils (table 5), 149 contained lymphocytes, and monocytes were found in 89 patient samples. There was no significant difference in percentages of alveolar macrophages, lymphocytes and monocytes in the asthma versus nonasthma group. The mean cell counts for monocytes, eosinophils, lymphocytes and neutrophils are presented for Chlamydia-positive versus -negative samples (DNA and culture) and asthma versus nonasthma subcohorts (table 5). The mean eosinophil count was significantly higher in asthmatics (5.5\%) versus nonasthmatics $(0.06 \% ; \mathrm{p}=0.001$, Fisher's exact test). The range of BALF eosinophil counts in asthmatic patients was $1-25 \%$. The mean neutrophil count in Chlamydia culture-positive patients was $68.39 \%$ compared to $30.87 \%$ in culture-negative subjects ( $p=0.001$, Fisher's exact test (two-tailed)). Since this group of children all had severe respiratory diseases and many had GERD, it was not surprising to discover that 175 out of the 182 BAL samples contained at least some LLMs. The cytological evaluation did not reveal a statistically significant association between the finding of LLMs and Chlamydia organisms. In total, 21 patients harboured few LLMs (1-3 per field) in their BALF, 66 patients had moderate numbers (4-6 per field) and 95 patients had many LLMs ( $\geqslant 6$ LLMs per field) in their BALF. Overall, 68 $(90 \%)$ out of 75 culture-positive versus 93 (87\%) out of 107 culture-negative patient samples displayed moderate to many LLM (table 5).

\section{DISCUSSION}

Chronic lung disease affects an increasingly wide cross-section of the world's population, manifesting itself mainly as asthma, 
TABLE 5 Bronchoalveolar lavage fluid cellularity and the presence of Chlamydia

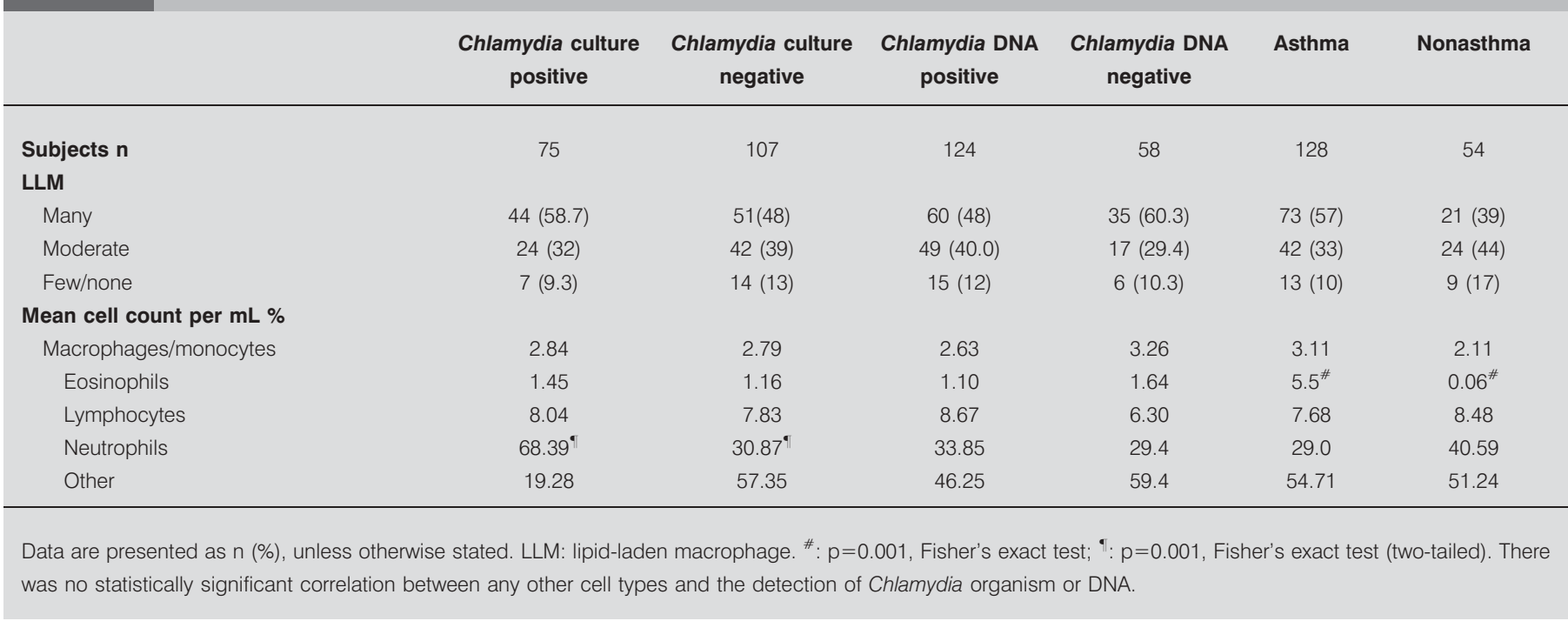

COPD, pneumonia and bronchitis [2, 18]. Globally, respiratory infections in childhood are a leading cause of disease and substantially contribute to school absence and severe economic strain on healthcare resources [30]. In the developing world, respiratory infections are also a major cause of childhood mortality [31]. Respiratory diseases of infancy and childhood are predominantly infectious in nature and can be caused by either viruses, bacteria or parasites [32].

In the current observational study it was demonstrated that human strains of Chlamydia, $C t$ and $C p$, can be isolated from the lungs of children with chronic respiratory disease. $C p$ has long been reported as an aetiological agent of community-acquired pneumonia and has been found in high prevalence in the lungs of adults [33], and also in the respiratory secretions of adult asthmatics, and lungs of COPD patients [34]. Recently, the present authors reported that $C p$ can also infect the lung tissue of children and might contribute to the pathology commonly seen in a variety of chronic respiratory diseases [22]. While the number of patients with chlamydial DNA in their lower respiratory tract analysed in the current study is surprisingly high compared with those in some recent studies $[35,36]$, the cohort is significantly larger. Furthermore, the type of samples collected differs from most published work to date, which utilises samples from the upper respiratory tract. It is also noteworthy that the present cohort is a select group of severely ill children with chronic respiratory disease and, as such, may not be reflective of the general population. Most other studies that report a prevalence of $C p$ in the $5-30 \%$ range assess sputum, nasal aspirates or throat swab samples $[35,37,38]$ as opposed to bronchial washings, a more invasive procedure with which samples were obtained in the current study. In a 2001 study where C $p$ PCR was performed on tracheobronchial aspirate, the authors reported a $51.9 \%$ prevalence was reported [39]. It should again be noted that the samples used in the present study were residual in nature and not obtained for the purpose of research, but as a part of the diagnostic evaluation of each patient. Importantly, most studies by others have only tested for the presence of $C p$ DNA, while the presence of both $C p$ and $C t$ DNA was assayed for by the current authors.
The data revealed a significant association between cultivable Chlamydia and asthma diagnosis, consistent with earlier findings. Importantly, the data also confirm that at earlier ages of life, $C t$ appears more prevalent than $C p$ in BALF samples. Although the organisms were also found in the neonate to 2 yrs old age group, it is not until age 5-10 yrs that an increased prevalence of $C p$ is observed. This suggests that these later infections may have been contracted through increased social interactions, possibly in pre-school or day care settings. These findings agree with previously published data, suggesting that $C t$ can be found in the lower respiratory tract of newborns and can lead to pneumonitis [12, 15, 21]. Evidence for the presence of $C t$ in the human placental tissue also exists [40-42].

Increased lipid content in alveolar macrophages of BALF is thought to be a useful indicator for recurrent pulmonary aspiration [43, 44]. Previous studies have confirmed that Chlamydia has the ability to survive, and even thrive, in alveolar macrophages $[45,46]$. It has been previously reported that $C p$ induces foam cell formation by human monocytederived macrophages [47]. Exposure of macrophages to $C p$, followed by the addition of low-density lipoprotein in tissue culture, caused a marked increase in the number of foam cells and accumulation of cholesteryl esters [47]. While not statistically significant, the current authors observed a correlation between the finding of moderate to many LLM (i.e. $>4$ cells per high power field) and Chlamydia positivity by PCR and culture. Infection of macrophages by Chlamydia, coupled with epithelial cell damage in the airways, could increase the inflammatory response in the lungs. With increased oxidative bursts by these phagocytes and the release of proinflammatory cytokines such as tumour necrosis factor- $\alpha$, interleukin (IL)-1 $\beta$, IL-6 and IL-8, airway hyperreactivity and pulmonary inflammation might be significantly increased.

In the current study, asthma patients treated with a combination of oral and inhaled corticosteroids were more likely to harbour chlamydial DNA (table 5). Inhaled glucocorticoids are a mainstay of asthma therapy. Oral steroid treatment is the most 
potent therapeutic intervention available for the effective relief of symptoms in acute and chronic asthma, especially for patients with severe disease. However, corticosteroids negatively affect many aspects of cell-mediated immunity and favour the shift from a T-helper type- 1 response towards a Thelper type- 2 response. Therefore, corticosteroids may severely impact the host's ability to eradicate an intracellular pathogen, such as Chlamydia, which requires properly functioning cellmediated (T-helper type-1) immune responses for pathogen clearance. Previous in vitro studies confirm that persistent Chlamydia in macrophages are reactivated when corticosteroid treatment is administered, resulting in the release of infectious elementary body particles into the immediate surroundings, whereby new cells are infected [48, 49]. Corticosteroids have also been shown to reactivate persistent Chlamydia carriage leading to an active growth phase, thus increasing the production of proinflammatory cytokines at the site of infection and further amplifying inflammation in the airways of patients with asthma [50-53]. The combination of both inhaled and oral steroid treatment might represent an increased amount of corticosteroids in the circulation leading to increased reactivation of Chlamydia. While antibiotic treatment of the patients in the present study was not attempted, previously published work by others has demonstrated that clarithromycin therapy improves lung function in subjects with positive PCR findings for Chlamydia and Mycoplasma [52, 54, 55].

Along with previously published studies, the present work suggests that further in-depth investigations of the involvement of the Chlamydiaceae family of obligate intracellular pathogens in the aetiology and exacerbation of asthma and other chronic respiratory diseases, particularly in paediatric populations, are needed. The current data confirms the presence of both Chlamydia trachomatis and Chlamydia pneumoniae organisms in the lungs of these patients. Importantly, patients with asthma and other inflammatory airway disease were more likely to harbour cultivable chlamydial organisms in their lower respiratory tract.

\section{ACKNOWLEDGEMENTS}

The authors wish to express their appreciation and thanks to the staff of the Transfusion Medicine Service and of the haematology laboratory at Baystate Medical Center (Springfield, MA, USA) for their kind support in this investigation.

\section{REFERENCES}

1 Cashat-Cruz M, Morales-Aguirre JJ, Mendoza-Azpiri M. Respiratory tract infections in children in developing countries. Semin Pediatr Infect Dis 2005; 16: 84-92.

2 World Health Organization. WHO Consultation on the development of a comprehensive approach to for the prevention and control of Chronic Respiratory diseases. Management of Noncommunicable Diseases Department, Chronic respiratory Disease and Arthritis. Geneva, World Health Organization, 2001. Available from: http://whqlibdoc. who.int/hq/2001/WHO_NMH_MNC_CRA_01.1.pdf

3 Colarizi P, Chiesa C, Pacifico L, et al. Chlamydia trachomatisassociated respiratory disease in the very early neonatal period. Acta Paediatr 1996; 85: 991-994.
4 Biscione GL, Corne J, Chauhan AJ, Johnston SL. Increased frequency of detection of Chlamydophila pneumoniae in asthma. Eur Respir J 2004; 24: 745-749.

5 Groenewegen KH, Wouters EF. Bacterial infections in patients requiring admission for an acute exacerbation of COPD; a 1-year prospective study. Respir Med 2003; 97: 770-777.

6 Karnak D, Beng-sun S, Beder S, Kayacan O. Chlamydia pneumoniae infection and acute exacerbation of chronic obstructive pulmonary disease (COPD). Respir Med 2001; 95: 811-816.

7 Hammerschlag MR. The role of Chlamydia in upper respiratory tract infections. Curr Infect Dis Rep 2000; 2: 115-120.

8 Tsai MH, Huang YC, Chen CJ, et al. Chlamydial pneumonia in children requiring hospitalization: effect of mixed infection on clinical outcome. J Microbiol Immunol Infect 2005; 38: 117-122.

9 Oba Y, Salzman G. Chlamydial pneumonias. Overview of infection with the three main chlamydial species that cause respiratory disease in humans. eMedicine, February 2007. Available from http://www.emedicine.com/med/ TOPIC341.HTM

10 Gern JE, Lemanske RF Jr. Infectious triggers of pediatric asthma. Pediatr Clin North Am 2003; 50, 555-575: vi.

11 Montalbano MM, Lemanske RF Jr. Infections and asthma in children. Curr Opin Pediatr 2002; 14: 334-337.

12 Numazaki K, Asanuma H, Niida Y. Chlamydia trachomatis infection in early neonatal period. BMC Infect Dis 2003; 3: 2.

13 Adderley-Kelly B, Stephens EM. Chlamydia: a major health threat to adolescents and young adults. ABNF J 2005; 16: 52-55.

$14 \mathrm{Wu} \mathrm{S}$, Shen L, Liu G. Study on vertical transmission of Chlamydia trachomatis using PCR and DNA sequencing. Chin Med J (Engl) 1999; 112: 396-399.

15 Darville T. Chlamydia trachomatis infections in neonates and young children. Semin Pediatr Infect Dis 2005; 16: 235-244.

16 McIntosh K. Community-acquired pneumonia in children. N Engl J Med 2002; 346: 429-437.

17 Herieka E, Dhar J. Acute neonatal respiratory failure and Chlamydia trachomatis. Sex Transm Infect 2001; 77: 135-136.

18 Acute respiratory infections: the forgotten pandemic. Communique from the International Conference on Acute Respiratory Infections, held in Canberra, Australia, 7-10 July 1997. Int J Tuberc Lung Dis 1998; 2: 2-4.

19 Ratelle S, Keno D, Hardwood M, Etkind PH. Neonatal chlamydial infections in Massachusetts, 1992-1993. Am J Prev Med 1997; 13: 221-224.

20 Bell TA, Stamm WE, Wang SP, Kuo CC, Holmes KK, Grayston JT. Chronic Chlamydia trachomatis infections in infants. JAMA 1992; 267: 400-402.

21 Bavastrelli M, Midulla M, Rossi D, Salzano M. Chlamydia trachomatis infection in children with wheezing simulating asthma. Lancet 1992; 339: 1174.

22 Webley WC, Salva PS, Andrzejewski C, et al. The bronchial lavage of pediatric patients with asthma contains infectious Chlamydia. Am J Respir Crit Care Med 2005; 171: 1083-1088.

23 Berhman RE, Kliegman R, Arvin AM, Nelson WE. Nelson Textbook of Pediatrics. 15th Edn. Amsterdam, Saunders, 1996. 
24 Salva PS, Theroux C, Schwartz D. Safety of endobronchial biopsy in 170 children with chronic respiratory symptoms. Thorax 2003; 58: 1058-1060.

25 Everett KD, Bush RM, Andersen AA. Emended description of the order Chlamydiales, proposal of Parachlamydiaceae fam. nov. and Simkaniaceae fam. nov., each containing one monotypic genus, revised taxonomy of the family Chlamydiaceae, including a new genus and five new species, and standards for the identification of organisms. Int J Syst Bacteriol 1999; 49: 415-440.

26 Gaydos CA, Quinn TC, Bobo LD, Eiden JJ. Similarity of Chlamydia pneumoniae strains in the variable domain IV region of the major outer membrane protein gene. Infect Immun 1992; 60: 5319-5323.

27 Jurstrand M, Falk L, Fredlund H, et al. Characterization of Chlamydia trachomatis omp1 genotypes among sexually transmitted disease patients in Sweden. J Clin Microbiol 2001; 39: 3915-3919.

28 Global Initiative for Asthma. Global Strategy for Asthma Management and Prevention, 2007 update. Available from: www.ginasthma.com/Guidelineitem.asp? $11=2 \& 12=1 \&$ intId $=60$

29 National Heart, Lung, and Blood Institutes and Global Initiative for Asthma. Global Strategy for Asthma Management and Prevention. NIH Publication 02-3659. Bethesda, National Institutes of Health, 2002.

30 Monto AS, Sullivan KM. Acute respiratory illness in the community. Frequency of illness and the agents involved. Epidemiol Infect 1993; 110: 145-160.

31 Berman S. Epidemiology of acute respiratory infections in children of developing countries. Rev Infect Dis 1991; 13: S454-S462.

32 Graham NM. The epidemiology of acute respiratory infections in children and adults: a global perspective. Epidemiol Rev 1990; 12: 149-178.

$33 \mathrm{Wu}$ JS, Lin JC, Chang FY. Chlamydia pneumoniae infection in community-acquired pneumonia in Taiwan. I Microbiol Immunol Infect 2000; 33: 34-38.

$34 \mathrm{Wu}$ L, Skinner SJ, Lambie N, Vuletic JC, Blasi F, Black PN. Immunohistochemical staining for Chlamydia pneumoniae is increased in lung tissue from subjects with chronic obstructive pulmonary disease. Am J Respir Crit Care Med 2000; 162: 1148-1151.

35 Teig N, Anders A, Schmidt C, Rieger C, Gatermann S. Chlamydophila pneumoniae and Mycoplasma pneumoniae in respiratory specimens of children with chronic lung diseases. Thorax 2005; 60: 962-966.

36 Schmidt SM, Muller CE, Krechting M, Wiersbitzky H, Gurtler L, Wiersbitzky SK. Chlamydia pneumoniae carriage and infection in hospitalized children with respiratory tract diseases. Infection 2003; 31: 410-416.

37 Cunningham AF, Johnston SL, Julious SA, Lampe FC, Ward ME. Chronic Chlamydia pneumoniae infection and asthma exacerbations in children. Eur Respir J 1998; 11: 345-349.
38 Esposito S, Principi N. Asthma in children: are Chlamydia or Mycoplasma involved? Paediatr Drugs 2001; 3: 159-168.

39 Schmidt SM, Muller CE, Bruns R, Wiersbitzky SK. Bronchial Chlamydia pneumoniae infection, markers of allergic inflammation and lung function in children. Pediatr Allergy Immunol 2001; 12: 257-265.

40 Numazaki K, Chiba S, Kogawa K, Umetsu M, Motoya H, Nakao T. Chronic respiratory disease in premature infants caused by Chlamydia trachomatis. J Clin Pathol 1986; 39: 84-88.

41 Gencay M, Puolakkainen M, Wahlstrom T, et al. Chlamydia trachomatis detected in human placenta. J Clin Pathol 1997; 50: 852-855.

42 Thorp JM Jr, Katz VL, Fowler LJ, Kurtzman JT, Bowes WA Jr. Fetal death from chlamydial infection across intact amniotic membranes. Am J Obstet Gynecol 1989; 161: 1245-1246.

43 Kazachkov MY, Muhlebach MS, Livasy CA, Noah TL. Lipid-laden macrophage index and inflammation in bronchoalveolar lavage fluids in children. Eur Respir J 2001; 18: 790-795.

44 Knauer-Fischer S, Ratjen F. Lipid-laden macrophages in bronchoalveolar lavage fluid as a marker for pulmonary aspiration. Pediatr Pulmonol 1999; 27: 419-422.

45 Haranaga S, Yamaguchi $H$, Ikejima $H$, Friedman $H$, Yamamoto Y. Chlamydia pneumoniae infection of alveolar macrophages: a model. J Infect Dis 2003; 187: 1107-1115.

46 Blasi F, Centanni S, Allegra L. Chlamydia pneumoniae: crossing the barriers? Eur Respir J 2004; 23: 499-500.

47 Kalayoglu MV, Byrne GI. Induction of macrophage foam cell formation by Chlamydia pneumoniae. J Infect Dis 1998; 177: 725-729.

48 Malinverni R, Kuo CC, Campbell LA, Grayston JT. Reactivation of Chlamydia pneumoniae lung infection in mice by cortisone. J Infect Dis 1995; 172: 593-594.

49 Cook PJ, Honeybourne D, Wise R, Davies P. Chlamydia pneumoniae antibody titres are significantly associated with the use of steroid medication in respiratory disease. Eur Respir J 1996; 9: Suppl. 23, 5s.

50 von Hertzen LC. Role of persistent infection in the control and severity of asthma: focus on Chlamydia pneumoniae. Eur Respir J 2002; 19: 546-556.

51 Black PN, Scicchitano R, Jenkins CR, et al. Serological evidence of infection with Chlamydia pneumoniae is related to the severity of asthma. Eur Respir J 2000; 15: 254-259.

52 Hahn DL, Bukstein D, Luskin A, Zeitz H. Evidence for Chlamydia pneumoniae infection in steroid-dependent asthma. Ann Allergy Asthma Immunol 1998; 80: 45-49.

53 Yang YS, Kuo CC, Chen WJ. Reactivation of Chlamydia trachomatis lung infection in mice by cortisone. Infect Immun 1983; 39: 655-658.

54 Kraft M, Cassell GH, Pak J, Martin RJ. Mycoplasma pneumoniae and Chlamydia pneumoniae in asthma: effect of clarithromycin. Chest 2002; 121: 1782-1788.

55 Hahn DL. Treatment of Chlamydia pneumoniae infection in adult asthma: a before-after trial. J Fam Pract 1995; 41: 345-351. 